\title{
A covid-19 Patient with Cavernous Sinus Thrombosis Post Dental Extraction a Diagnostic Dilemma
}

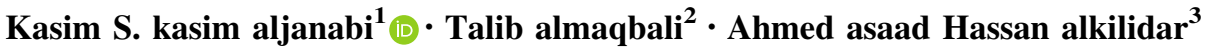

Received: 21 December 2020/ Accepted: 8 February 2021 / Published online: 26 February 2021

(C) Association of Otolaryngologists of India 2021

\begin{abstract}
Prior to the antibiotics era, the mortality rate from cavernous sinus thrombosis (CST) was very high reaching $100 \%$. There have been very few reports of CST associated with tooth extraction on the same time Coronavirus disease of 2019 (COVID-19) is known to increase the risk of developing venous thromboembolism; therefore, patients with COVID-19 may present with cerebral venous sinus thrombosis. We present a case with diagnostic dilemma and first to be reported in the literature.
\end{abstract}

Keywords Cavernous sinus thrombosis · Odontogenic ·

Tooth extraction $\cdot$ Covid -19

\section{Introduction}

Cavernous sinus thrombosis (CST) was first described by Bright in 1831 as a complication of epidural and subdural contamination [1]. It has complex neurovascular anatomic relationships; therefore CST is the most complicated type of intracranial septic thrombosis [2]. The incidence of CST has always been low, with only approximately 200 case reports found in literature. The mortality rate from CST was effectively $100 \%$ prior to antibiotics era and usually is due to sepsis or central nervous system infection. With aggressive management, the mortality rate is reduced to $30 \%$ [3].

Kasim S. kasim aljanabi

kasims79@yahoo.com

ENT Specialist, Sohar hospital, Sohar, Oman

Consultant ENT, Sohar hospital, Sohar, Oman

3 Senior Specialist, Sohar hospital, Sohar, Oman
There are very few reports of odontogenic CST identified post tooth extraction [4]. CST can result from infection of the paranasal sinuses or any of the anatomic structures drained by the cavernous sinus, including the mid-face, orbit, and oral cavity [5]. And recently with the Covid19infection there is increasing reports with cerebrovenous thrombosis as complications of the disease so we present a diagnostic dilemma of CST soon after dental extraction with a covid-19 positive patient.

\section{Case Report}

Fifty two years old gentleman not known to have chronic illnesses, he recovered uneventfully from mild Covid-19 infection three months back. Patient presented to the emergency department with progressive facial swelling, reduce vision and diplopia for three days duration. There was history of teeth extraction seven days prior to that, involving the left and right upper 1st molars. The teeth extraction was uneventful until he noticed progressive facial swelling involving the left side first then progress to involve the whole midface with eyes proptosis and reduced vision. There were no respiratory symptoms. The patient general condition was toxic with high grade fever. Upon examination there was defuse mid face swelling with bilateral orbital proptosis, chemosis and restricted eye mobility at all gazes. The left eye there were no perception of light with pupil noted fixed and dilated. The right side pupil was noted to have sluggish reaction to light. Oral exam revealed restricted mouth opening secondary to facial swelling dental sockets exam revealed no local infection hard and soft palate were normal apart from soft palate edema. Blood investigations showed Covid-19 positive, elevated WBC 30.000 cubic millimeter with predominantly 
neutrophilia his C-reactive protein was above $300 \mathrm{mg} / \mathrm{l}$ and his blood sugar was normal initially but subsequently was deranged secondary to severe sepsis. A clinical impression of cavernous sinus thrombosis was reached and patient was approached with multi-disciplinary team approach involving (ENT, Ophthalmology, Dental, Neurosurgery, medicine and ID team). Due to significant soft palate and upper airway edema, patient was reporting increasing difficulty in breathing and had to be intubated within $24 \mathrm{~h}$ of admission. A confirmation of cavernous sinus thrombosis was reached with Contrast enhanced computed tomography (CT) scans and cerebral CTangio (Figs. 1, 2 and 3) revealed poor enhancement of both cavernous sinuses denoting acute thrombosis. Rests of dural sinuses are patent and fully enhanced. The anterior (ACA, MCA) and posterior (Vertebral, Basilar and PCA) cerebral arterial circulation are fully enhanced with normal anastomosis. There is no enhancement of both superior ophthalmic veins which appear dilated proximally with hypo density seen represent thrombosis. Incidental bilateral mucosal thickening of both maxillary sinuses, ethmoid air cells and sphenoid sinus with sinonasal polyposis. CCA and ICA are enhanced and patent on both sides. MRV was not done as patient was not fit to be transferred to another facility for MRI. Nasal endoscopic exam revealed normal mucosal color with bilateral grade II nasal polys no evidence of mucin or pus and no blackish or pale mucosa noted. Multiple biopsies from the polyps and inferior turbinate on both sides were obtained. Histopathological exam showed no evidence of invasive fungal infection.
On the clinical and radiological evidences a provisional diagnosis of cavernous sinus thrombosis secondary to dental extraction was made and the patient was treated aggressively with high dose triple antibiotics (Meropenem $500 \mathrm{mg}$ bid, Rocephin $2 \mathrm{~g}$ tid and Vancomycin $1.5 \mathrm{~g}$ bid), high dose of heparin (8000 iu od) and steroids. Unfortunately his condition deteriorate very fast within $72 \mathrm{~h}$ and he succumbed of the disease complications.

\section{Discussion}

The cavernous sinus (CS) is a dural venous sinus situated lateral to the sella turcica, in between the two layers of the dura, in the middle cranial fossa. Within the cavernous sinus runs the third, fourth, and sixth cranial nerves; ophthalmic and maxillary divisions of the fifth cranial nerve; and internal carotid artery with its surrounding sympathetic plexus. The CS drains blood from the orbit through ophthalmic veins and from the face through the facial veins via pterygoid plexus then the blood from CS empty into both the superior and inferior petrosal sinuses and ultimately into the internal jugular veins. Blood can flow in either direction, as these complex webs of valveless veins. Facial infections involving the nose, tonsils, and orbits can easily spread by this complex pathway to the cavernous sinus [6]. According to Shaw [7], various routes of infection that can lead to septic thrombosis which are: 1-from the face through the angular and ophthalmic veins, 2- from the middle ear through the superior petrousal sinus, 3- from the
Fig. 1 Axial CT angiography showing normal enhacement of cavernous carotid artery(red arrows) with thrombosed and dilated superior ophthalmic veins(black arrows)

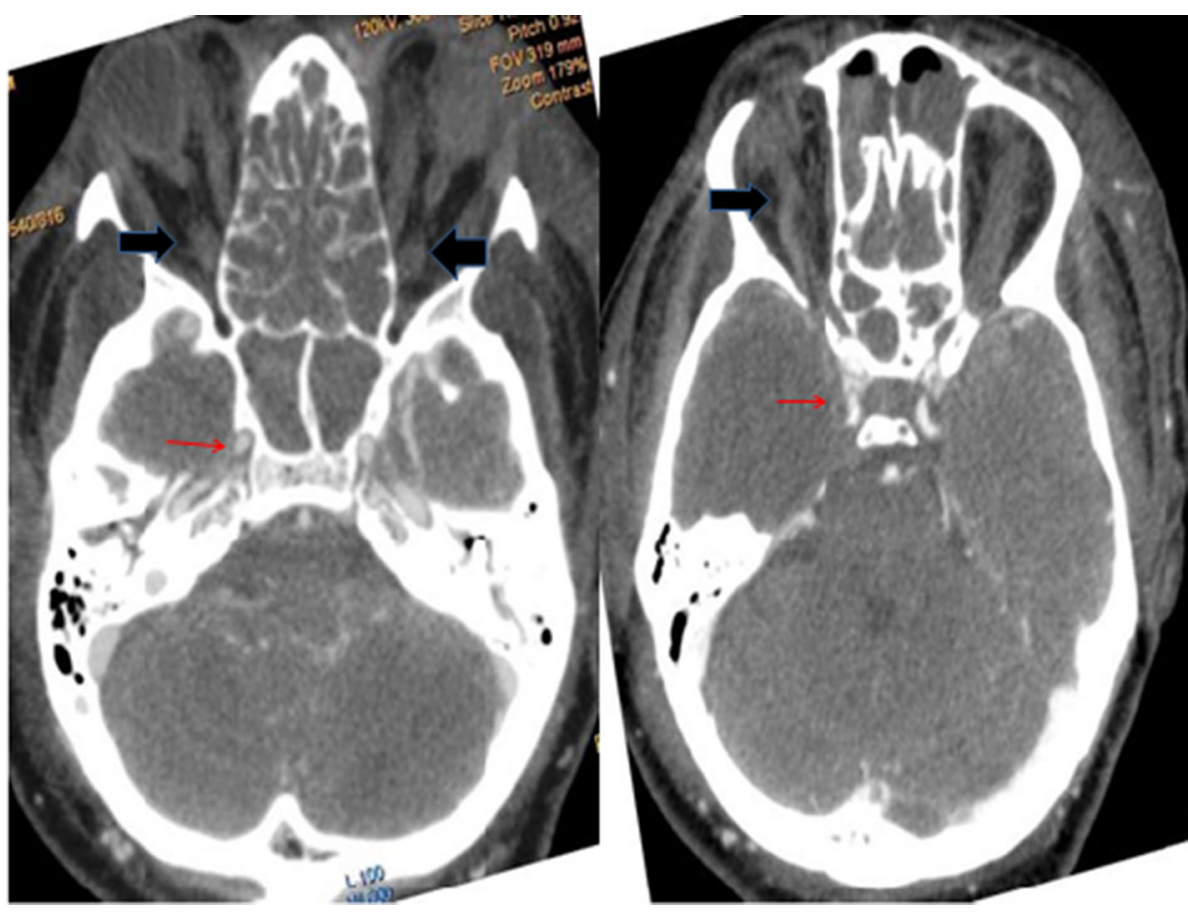


Fig. 2 Axial CT venography showing cavernous sinus thrombosis

Fig. 3 Reconstructive 3d image showing cavernous sinus thrombosis with patent carotid artery system
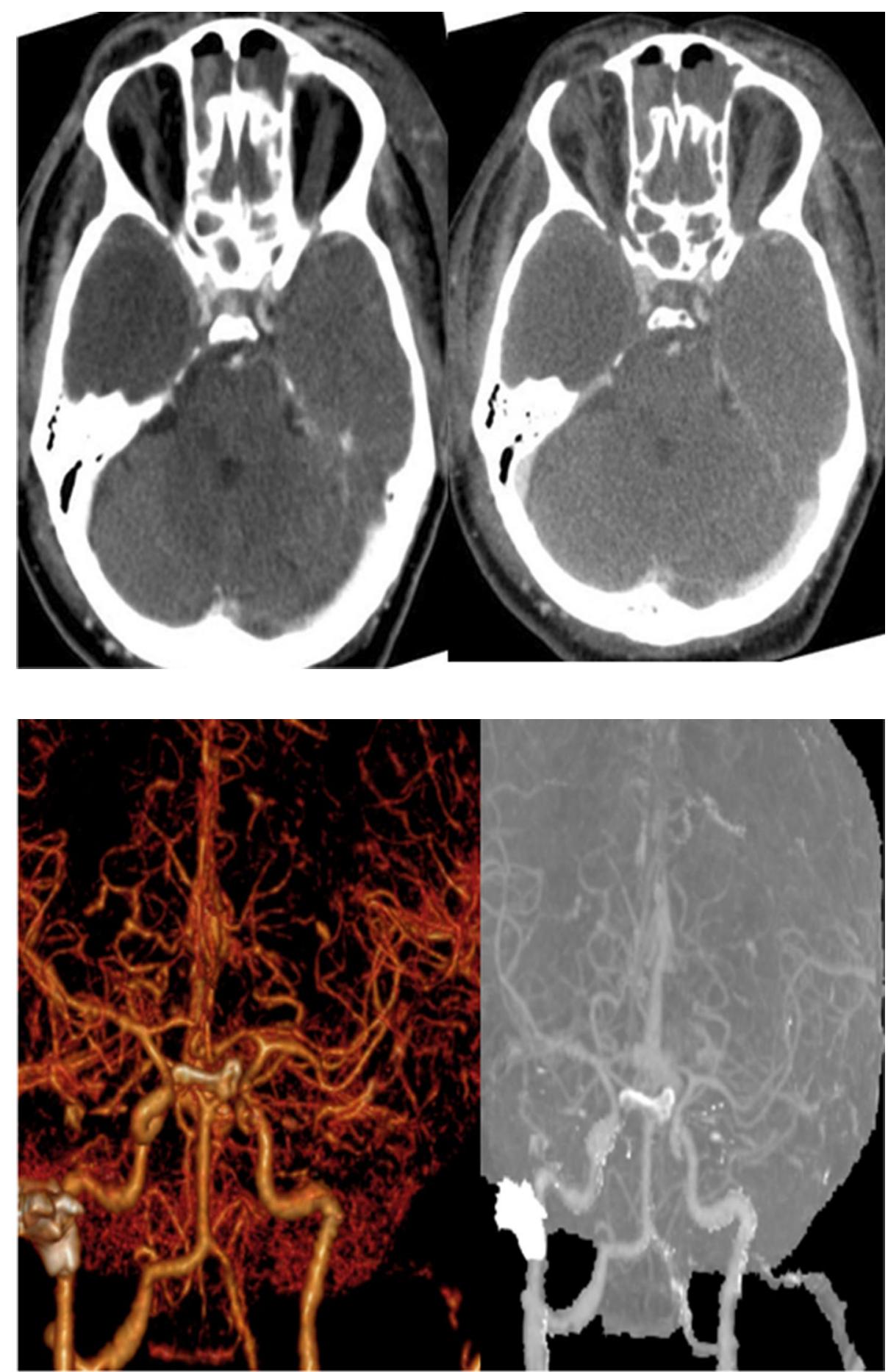

teeth, maxillary sinus, and cervical vertebrae through the pterygoid plexus emptying into the inferior ophthalmic vein 4- from the sphenoid sinus by direct extension or draining emissary veins, 5- by extension from an infected internal jugular vein, lateral sinus, or petrosal sinus, or 6from the plexus of veins surrounding the internal carotid artery. The spread of infection in our case was likely from the pterygoid plexus to the inferior ophthalmic vein to the cavernous sinus. It is estimated that around $7 \%$ of septic CST is of dental origin [8]. It was suggested that the infection is probably occurs when the pterygoid plexus is contaminated by a needle incorrectly inserted during the posterior superior alveolar nerve block [9] which might be the cause of CST in our case as there was no local dental infection or collection at the extraction sites. However our patient had another possible cause (novel Severe Acute 
Respiratory Syndrome-coronavirus 2 (SARS-CoV2). A novel Severe Acute Respiratory Syndrome-coronavirus 2 (SARS-CoV2) was identified on January 7, 2020, by the Chinese Center for Disease Control and Prevention from a nasopharyngeal swab in a patient with atypical pneumonia in Wuhan, Hubei province, China [10]. The mortality rate of critically ill patients with COVID-19 is high as of 1 st November 2020 more than 46 million tested positive of COVID-19 and total deaths reached to 1.2 million worldwide.

Recent studies have reported the potential development of a hypercoagulable state in COVID-19 [11-14]. Postulated causes are generation and inhibition of fibrinolysis [15]. Moreover, hypoxemia is associated with an elevation of blood viscosity and activation of hypoxia-related genes that mediate coagulation and fibrinolysis, favoring thrombotic events $[16,17]$. This septic-like coagulopathy additionally may lead to venous thrombosis, pulmonary embolism, and ultimately disseminated intravascular coagulation [15]. Cerebral venous thrombosis, in particular, can present with a broad variety of neurologic signs and symptoms. The superior sagittal sinus is the most frequently reported cerebral venous structure involved (63\% of patients in a large MR imaging series); however, more extensive multi-site thrombosis is the typical finding [17-19]. Therefore our case provides a diagnostic challenge and dilemma whether the cause of cavernous sinus thrombosis caused by dental infection or the COVID-19 sequel. In conclusion the unusual presentation with tragic cerebral venous thrombosis in previously healthy patient infected with SARS-CoV-2 was a high possibility and these cases provide hints as to the accumulating evidence that COVID-19 is a serious contributor to hypercoagulation, increasing the fatality of the disease. Heightened awareness of this atypical but potentially treatable complication of the COVID-19 disease spectrum is encouraged.

Acknowledgements Special thanks to all the teams helped in giving the care and treatment for the this patient (ENT, Ophthalmology, Dental, Neurosurgery, medicine and ID team) and special appreciation and gratitude to all frontline workers on covid-19 pandemic.

\section{Compliance with Ethical Standards}

Conflict of interest As a corresponding author and on behalf of coauthors, I declared that I take full responsibility for the integrity of the content of the manuscript. There is no conflict of interest needed to be declared. There is no financial support received from any organization. Consent was taken from the patient family.

\section{References}

1. Tempea V, Gorun G (1959) Cavernous sinus thrombosis. AMA Arch Otolaryngol 69:220-223
2. Laupland KB (2007) Vascular and parameningeal infections of the head and neck. Infect Dis Clin North Am 21:577

3. Absoud M, Hikmet F, Dey P, Joffe M, Thambapillai E (2006) Bilateral cavernous sinus thrombosis complicating sinusitis. J R Soc Med 99:474-476

4. Colbert S, Cameron M, Williams J (2011) Septic thrombosis of the cavernous sinus and dental infection. Br J Oral MaxillofacSurg 49:e25-e26

5. Pavlovich P, Looi A, Rootman J (2006) Septic thrombosis of the cavernous sinus: two different mechanisms. Orbit 25:39-43

6. S Standring; NR. Borley, et al (eds) (2008) Gray's anatomy: the anatomical basis of clinical practice, 40th edn. Churchill Livingstone, London

7. Shaw RE (1952) Cavernous sinus thrombophlebitis: a review. $\mathrm{Br}$ J Surg 40:40-48

8. Alwraikat AA, Alawneh HI (2010) Cavernoussinusthrombosis as a fatal complication of a dental abscess: a case report. J Royal Med Serv 17:20-23

9. Prabhu S, Jain SK, Dal Singh V (2015) Cavernous sinus thrombophlebitis (sans thrombosis) secondary to odontogenicfascial space infection: an uncommon complication with unusual presentation. J Maxillofac Oral Surg 14(Suppl 1):168-172

10. Chen N, Zhou M, Dong X et al (2020) Epidemiological and clinical characteristics of 99 cases of 2019 novel coronavirus pneumonia in Wuhan, China: a descriptive study. Lancet 395:507-13

11. Li Y, Wang M, Zhou Y, et al. (2020) Acute cerebrovascular disease following COVID-19: a single center, retrospective, observational study. SSRN Electron J. January 2020. https://www.researchgate.net/publication/340154622_Acute_Cer ebrovascular_Disease_FollowingCOVID-19_A_Single_Center_ Retrospective_Observational_Study. Accessed Apr 132020

12. WangW, Sun Q, Bao Y, et al. (2020) Analysis of risk factors for the thromboembolic events from 88 patients with COVID-19 pneumonia in Wuhan, China: a retrospective report. Lancet 2020 April 6. https://papers.ssrn.com/sol3/papers.cfm?abstract_id= 3559633. Accessed Apr 132020

13. Han H, Yang L, Liu R et al (2020) Prominent changes in blood coagulation of patients with SARS-CoV-2 infection. ClinChem Lab Med 7:1116-1120 ([Epub ahead of Print] CrossRef Medline)

14. Zhang Y, Xiao M, Zhang S et al (2020) Coagulopathy and antiphospholipid antibodies in patients with Covid-19. N Engl J Med 382:38 (CrossRef Medline)

15. Uthman IW, Gharavi AE (2002) Viral infections and antiphospholipid antibodies. Semin Arthritis Rheum 31:256-63 (CrossRef Medline)

16. Gupta N, Zhao YY, Evans CE (2019) The stimulation of thrombosis by hypoxia. Thromb Res 181:77-83 (CrossRef Medline)

17. Ortel TL (2010) Acquired thrombotic risk factors in the critical care setting. Crit Care Med 38:S43-50 (CrossRef Medline)

18. Canhao P, Ferro JM, Lindgren AG et al (2005) ISCVT investigators. Causes and predictors of death in cerebral venous thrombosis. Stroke 36:1720-25

19. Girot M, Ferro JM, Canhao P et al (2007) ISCVT investigators. Predictors of outcome in patients with cerebral venous thrombosis and intracerebral hemorrhage. Stroke 38:337-42

Publisher's Note Springer Nature remains neutral with regard to jurisdictional claims in published maps and institutional affiliations. 